\title{
Study on acoustic impedance conversion using an optimal chirplet analyzed in chirp SBP raw data
}

\author{
Chanho Park ${ }^{1} \cdot$ Wonsik Kim ${ }^{1} \cdot$ Jungkyun Shin ${ }^{1} \cdot$ Hyundo Kim $^{2} \cdot$ Joo Bong Jeong ${ }^{1}$
}

Received: 23 May 2018 / Accepted: 10 January 2019 / Published online: 26 February 2019

(c) The Author(s) 2019

\begin{abstract}
Chirplets are wavelets that are transmitted by a chirp sub-bottom profiler (SBP) system. They contain multiple frequencies, typically in the $\mathrm{kHz}$ range, and are modulated linearly within a fixed signal length, which makes efficient data analysis possible. However, when chirp SBP field data are recorded, they are usually not raw but have gone through a matching filter and been converted to a Klauder wavelet section or signal envelope. When pre-processed data are reviewed, anomalies can easily be distinguished by sight, and since the data are not raw, reliable quantitative analysis of physical properties is unlikely. Moreover, as chirplets are transmitted through media, their waveforms often become distorted, creating many difficulties in performing analysis of physical properties following data processing. In this study, raw data were used as input to perform an accurate physical analysis of the background media and anomalies at the site of a 600-year-old shipwreck off the coast of Korea. During data processing, an optimal chirplet found through an analysis of the seabed reflection in the raw data was used, instead of the original chirplet set that was applied during acquisition. Conventional data processing was performed in addition to sparse-spike deconvolution and acoustic impedance conversion, using the optimal chirplet found in the raw data. The acoustic impedance results obtained by using the optimal chirplet had a higher level of accuracy when determining the properties of the medium and identifying anomalies, compared to those obtained when the original chirplet was used.
\end{abstract}

Keywords Chirp SBP · Optimal chirplet · Sparse-spike deconvolution · Acoustic impedance

\section{Introduction}

Chirplets are a particular type of seismic wavelet that is transmitted in a chirp SBP (sub-bottom profiler) system (Mann and Haykin 1995). Unlike normal wavelets, chirplets consist of multiple frequencies above several $\mathrm{kHz}$ that are modulated within a fixed signal length. They generally use a tapering function to reduce the magnitude of the side-lobe that is a cause of reduced data quality (Baradello 2014). Furthermore, when cross-correlated with raw data, chirplets can produce Klauder wavelet sections, which have a narrow reflection surface and high temporal resolution.

Because of these characteristics, anomalies in chirp SBP data can usually be distinguished by sight and analyzed

Wonsik Kim

hyojin@kigam.re.kr

1 Pohang Branch, Korea Institute of Geoscience and Mineral Resources, Pohang, Republic of Korea

2 Geoview Ltd., Busan, Republic of Korea without any special data processing in the field. When chirp SBP data are acquired, they are usually not in the form of raw data, which preserves material properties, but instead, have gone through a matching filter and been converted to a Klauder wavelet section and/or signal envelope section.

The reported goals of most studies have not been quantitative analysis of material properties, but either classification of seabed sediments (LeBlanc et al. 1992; Stevenson et al. 2002) or increasing resolution (Baradello 2014; Gutowski et al. 2008; Henkart 2006; Quinn et al. 1998; Schwamborn et al. 2002). Few studies have attempted quantitative analysis of material properties. Bull et al. (1998) estimated the reflection coefficient of shipwrecks buried in the seabed through artificial models (acoustic impedance and reflection coefficient models) by mixing adjacent traces of raw data and then using the calculated polarity values and the mean amplitude values of each trace. However, their study was limited because it estimated the material properties of anomalies through an artificial model rather than by directly calculating the reflection coefficients of anomalies. 
Unlike existing studies, this study aims to directly reveal the physical properties of media and anomalies through conventional processing of raw data, as well as sparse-spike deconvolution and acoustic impedance conversion. In addition, this study attempts a new approach, which uses an optimal chirplet found from analysis of the seabed reflection of the raw data, rather than the original chirplet set derived during acquisition. The reason for this approach is that the signal length and frequency range of the original chirplet set during acquisition is expected to change considerably, as it is transmitted through the media, which is expected to affect the ultimate acoustic impedance results.

The raw chirp SBP data used in this study cover an ancient shipwreck, as well as relics within and outside the wreck. The methodology of this study was verified by comparing the acoustic impedance data obtained via the optimal chirplet and that obtained using the original chirplet. Further validation was achieved by comparing the shipwreck area's acoustic impedance results to the acoustic impedance results of a study area in which iron pots and ceramics were deliberately buried.

\section{Optimal chirplet analysis technique}

The name "chirp" refers to all signals in which frequencies change linearly, and a "chirplet" is a portmanteau of "chirp" and "wavelet," as it is a wavelet which is transmitted in a chirp SBP system. Chirplets are signals in which the frequency is modulated within a fixed signal length; they usually employ a tapering function (i.e. envelope function) so that a thin reflection surface with good resolution can be obtained when they are converted to a Klauder wavelet section. Here, a Klauder wavelet is a waveform that is created when autocorrelation is performed on a chirplet, while a Klauder wavelet section is a section that is created when cross-correlation is performed on the raw data and the chirplet.

Chirplets are high frequency signals (above several $\mathrm{kHz}$ ), and compared to lower frequency sound sources, are very vulnerable to noise and environmental changes. Consequently, the waveforms of chirplets often become distorted as they are transmitted through media. Moreover, chirplets must be maintained at a fixed signal length as they are transmitted through media, but can often become longer. For these reasons, chirplet frequency ranges and signal lengths that are suitable for the exploration data must be used in data processing. Gutowski et al. (2002) performed a study on data processing results according to a variety of different chirplet signatures and found that knowledge of the relevant chirplet signature is important.

In the current study, conventional data processing was performed in addition to a new form of data processing. The additional data processing included the creation of an optimal chirplet, sparse-spike deconvolution, and acoustic impedance conversion (Fig. 1). A detailed description of normal, conventional data processing has been omitted from this study; a good overview can be found in Kim (2014), Kim et al. (2016) and Schwamborn et al. (2002). This section provides a detailed description of the analysis technique for creating the optimal chirplet.
Fig. 1 Workflow of the data processing used in the study

\section{Existing data processing}

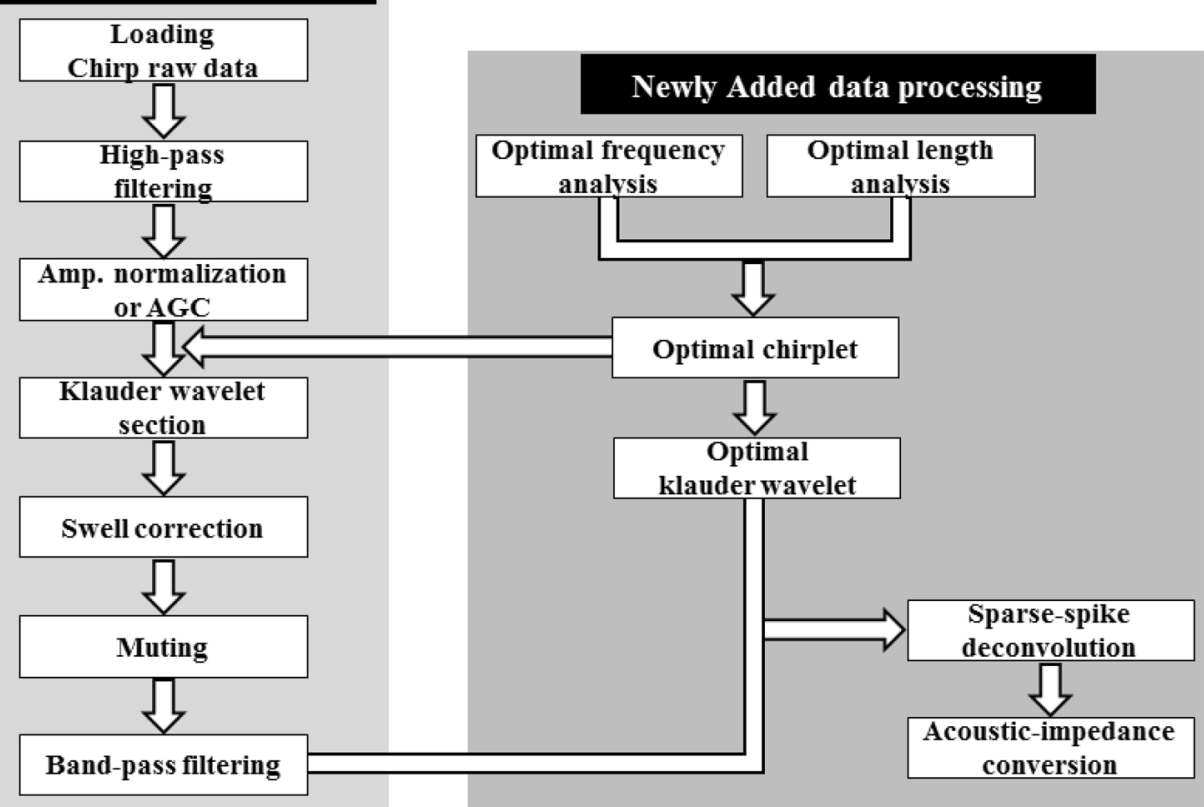


Most marine seismic exploration surveys record a signal in the form of a far-field signature and perform data processing to provide accurate results. However, most chirp SBP exploration is zero-offset exploration, and it uses high frequencies above several $\mathrm{kHz}$, making it possible to use seabed waveforms which are close to the far-field signature. Therefore, in this study of optimal chirplet analysis, the frequency range (minimum and maximum) and signal length were found from the raw data's seabed reflection signal. The analysis was performed with the following procedure.

First, the analysis range of the raw data's seabed reflection waves was set and recorded. Traces that were affected by anomalies (reflection, diffraction, etc.) were excluded from the analysis since incorrect analysis can result from their inclusion.

Second, the analysis range of the chirplet signature variables was set. The chirplet signature variables include the frequency range and the signal length. Suitable results can be obtained if the analysis range is set at approximately $80-120 \%$ of the original chirplet signature variable that was set during acquisition. For example, if the original chirplet's frequency range was $2000-7000 \mathrm{~Hz}$, the minimum frequency analysis range in this study was $1600-2400 \mathrm{~Hz}$, and the maximum frequency analysis range was $5600-8400 \mathrm{~Hz}$.

Third, a cross-correlation was performed between the many artificial chirplets, which incorporate the chirplet signature within the analysis range, and the seabed reflection section; the maximum instantaneous energy intensity values caused by each chirplet signature analysis variable were found. This shows that the variable that produces the maximum instantaneous energy intensity value is the chirplet signature's optimal variable. In the cross-correlation, if the two signals match well, the correlation becomes higher and the value becomes larger. At this point, if only values resulting from the cross-correlation are used, there can be multiple maximum values. Therefore, in the final analysis stage, it is beneficial to examine the instantaneous energy intensity within a signal envelope created by a Hilbert transform.

\section{Data acquisition and processing}

In this study, raw data, which preserves the form and physical properties of the chirplets, were obtained for analysis and used in determining the optimal chirplet. The raw chirp SBP data came from a survey of a ship that sank 600 years ago (1410-1420 CE) in the waters off Mado Island, in Korea's western sea.

The sea near Mado Island is part of a rias coast, so the water depth is shallow at 5-30 m, and has the characteristics of a very soft sediment environment. Moreover, the sea near Mado Island has fast currents, so it is a region where many ships have sunk throughout history (Fig. 2). The star symbol

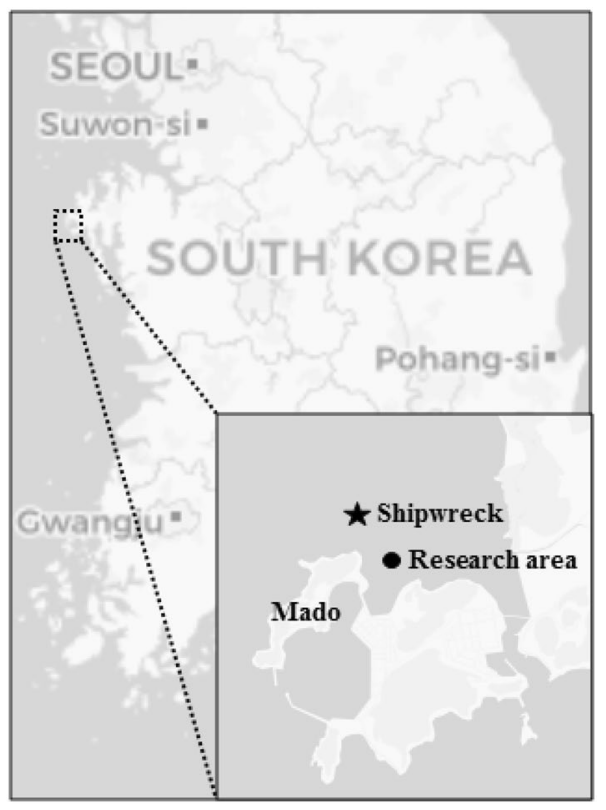

Fig. 2 Chirp SBP's exploration area map (star symbol=shipwreck area; circle symbol = study area)

in Fig. 2 refers to the shipwreck, and the circle symbol is the study region where the iron pots and ceramics relics were artificially buried; both areas verify the acoustic impedance results from the shipwreck area.

Before the seismic survey of the shipwreck area was performed, the shipwreck and the many relics (iron pots, ceramics, grains, etc.), which exist both inside and outside the wreck, were examined directly by divers (Fig. 3). In addition, chirp data acquisition was carried out after a test pit was performed in order to view the body of the ship and the relics. Here, a test pit refers to an operation in which the sediment on top of the shipwreck is skimmed off, and thereby exposing the shipwreck was exposed on the surface of the seabed for the relics investigation. The original chirplet signature used in the shipwreck area's chirp SBP exploration had a frequency range of $2000-7000 \mathrm{~Hz}$, a signal length of $5 \mathrm{~ms}$, a taper length of $2.5 \mathrm{~ms}$, and a sampling interval of 13 $\mu \mathrm{s}$. Figure 4 shows the raw chirp SBP data for the shipwreck area. The dotted rectangle shows the location of anomalies that can be distinguished by sight.

\section{Creation of optimal chirplet and comparison with original chirplet}

In this study, analysis was performed on traces 1-40 (which did not incorporate the effects of anomalies) in the preprocessed on-site data, and the arithmetic mean of the analysis values was calculated. Figure 5 shows a collection of mean values for the instantaneous energy intensity in the analysis 

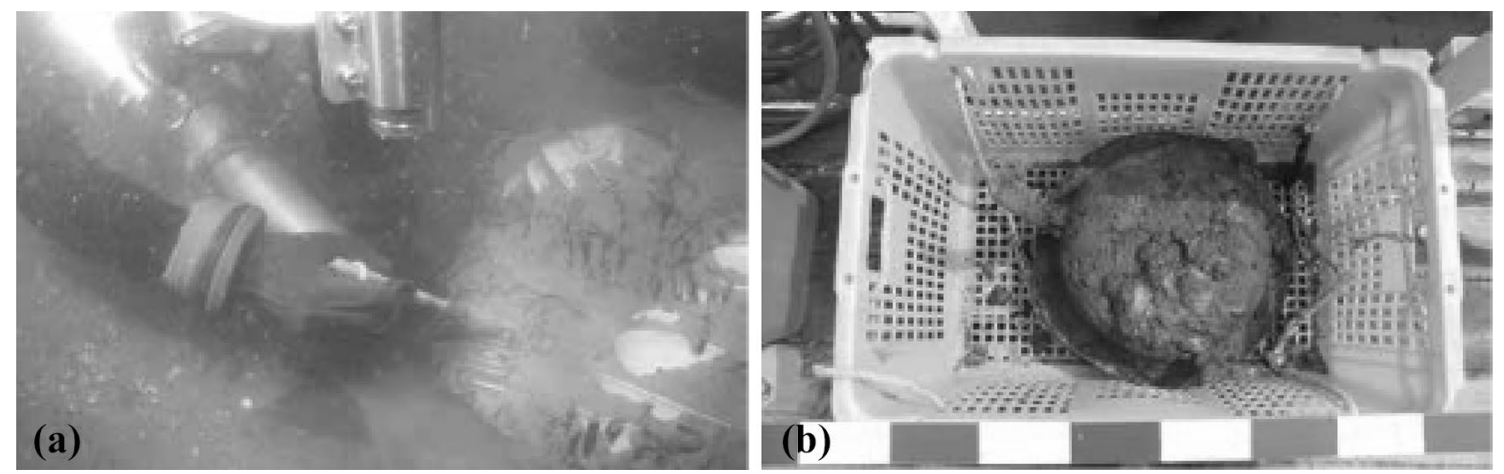

Fig. 3 a Ceramics and $\mathbf{b}$ excavated iron pots confirmed directly by divers inside and outside the shipwreck

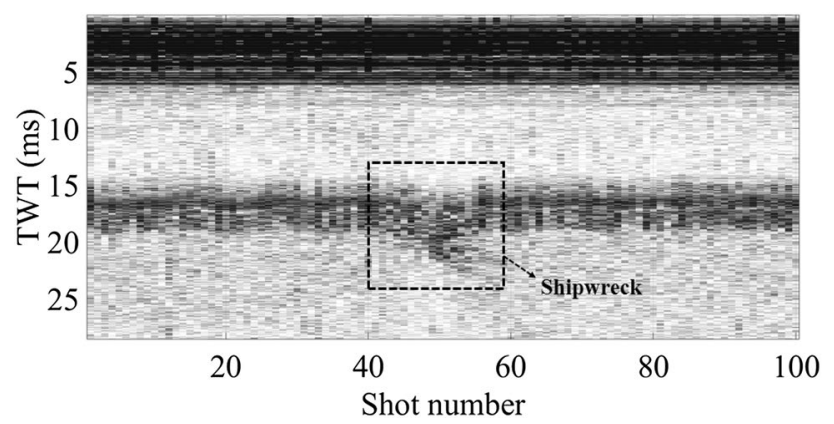

Fig. 4 Chirp SBP's raw data section. The dotted rectangle shows the location of the shipwreck

range of the 40 analyzed traces. The chirplet signature variable's analysis range was set to be wide in this instance to demonstrate the method. The maximum value of the instantaneous energy intensity appeared close to the shape of the envelope, and therefore, the maximum value could be easily distinguished.

The results of examining the variable values at the point where the maximum instantaneous energy intensity value occurred, show that there was a minimum frequency of $1811 \mathrm{~Hz}$, a maximum frequency of $7935 \mathrm{~Hz}$, and a signal length of $5.8 \mathrm{~ms}$. This means that the frequency range was wider and the signal length longer than the original chirplet signature that was defined during acquisition (Table 1).

The optimal chirplet signature that was found this way was used to create the optimal chirplet and the optimal Klauder wavelet. The optimal chirplet's taper length was set at half of the signal length, just as in the original chirplet (Table 1).

\section{Comparison of original and optimal chirplets}

Figure 6 shows a comparison of the original and optimal chirplets and Klauder wavelets. In this section, a comparison and analysis of the Klauder wavelet is made by performing
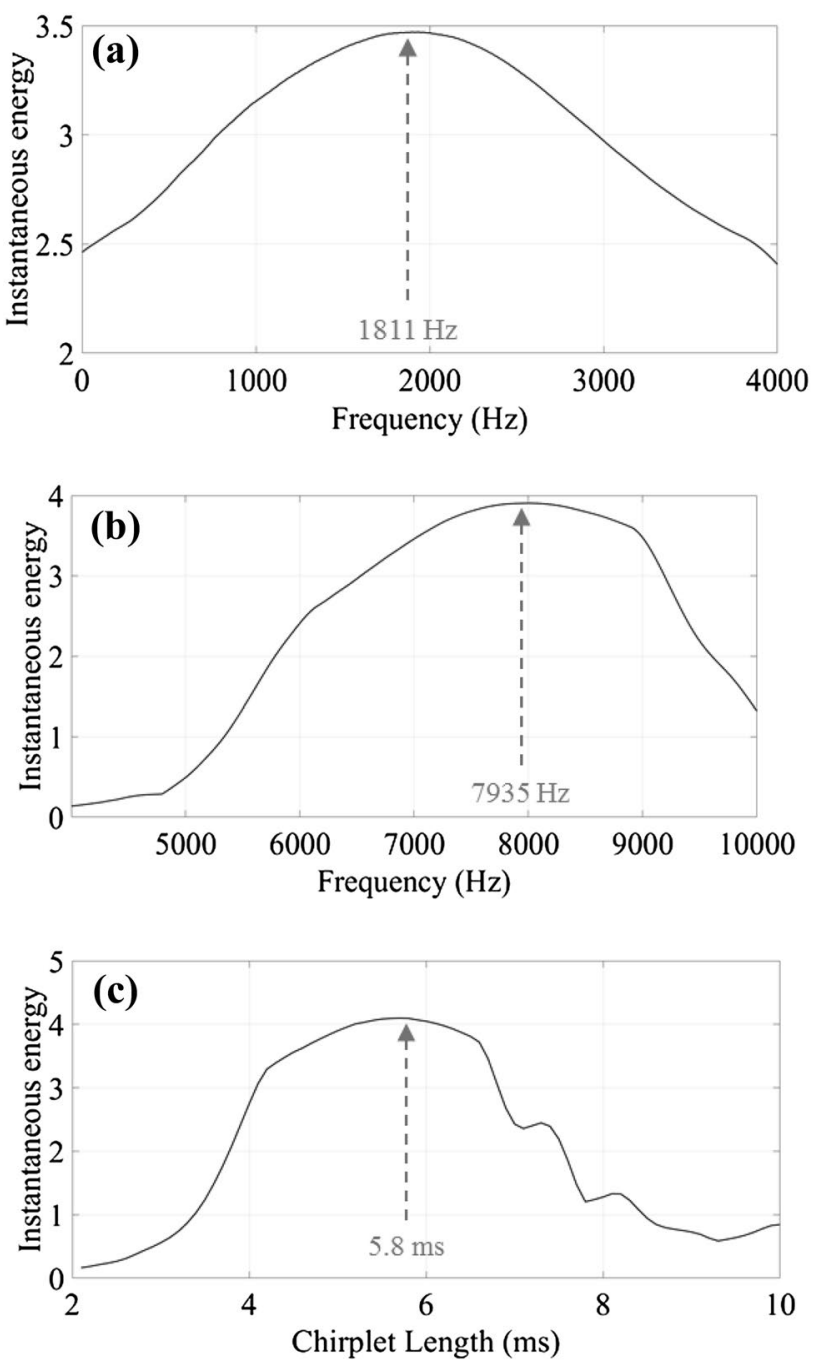

Fig. 5 Chirplet signature's instantaneous energy analysis results: a minimum frequency, b maximum frequency, and c chirplet's signal length 
Table 1 Original and optimal chirplet signatures

\begin{tabular}{lll}
\hline & Original chirplet & Optimal chirplet \\
\hline Pulse bandwidth & $2000-7000 \mathrm{~Hz}$ & $1811-7935 \mathrm{~Hz}$ \\
Pulse length & $5 \mathrm{~ms}$ & $5.8 \mathrm{~ms}$ \\
Pulse taper length & $2.5 \mathrm{~ms}$ & $2.9 \mathrm{~ms}$ \\
Sampling interval & $13 \mu \mathrm{s}$ & $13 \mu \mathrm{s}$ \\
\hline
\end{tabular}

a sensitivity analysis on the results when the original and optimal wavelets are used. In this study, the lobe that shows the maximum amplitude at the Klauder wavelet's $0 \mathrm{~ms}$ time period is called the main lobe, and the lobe which occurs on the side is called the side lobe or the secondary side lobe (Fig. 6).

The original Klauder wavelet's main lobe amplitude was 44\% larger than the side lobe's amplitude, and 92\% larger than the secondary side lobe's amplitude. In comparison, the optimal Klauder wavelet's main lobe amplitude was almost 48\% larger than the side lobe's amplitude, and 95\% larger than the secondary side lobe's amplitude, which shows an increase in the percentage difference relative to the original Klauder wavelet.

In addition, the optimal Klauder wavelet's main lobe amplitude was nearly $16 \%$ larger than the original Klauder wavelet's, while its side lobe amplitude was $0.2 \%$ smaller, and its secondary side lobe amplitude was $49 \%$ smaller.
Furthermore, the time interval between both side lobes in the optimal Klauder wavelet was almost $12 \%$ shorter than the original Klauder wavelet. These results suggest that the optimal Klauder wavelet will show more spiked waveforms than the original Klauder wavelet, which will have a direct effect on the data's resolution; because of this procedure, the optimal Klauder wavelet can find the location and reflection coefficient strength of anomalies and media more accurately than the original Klauder wavelet.

\section{Al conversion from reflection coefficients}

To find the optimal Klauder wavelet section (Fig. 7), the optimal Klauder wavelet was used to perform sparse-spike deconvolution, and then acoustic impedance conversion was performed.

\section{Sparse-spike deconvolution}

The sparse-spike deconvolution used in this study is an iterative process which uses the L1-norm to find the spike train that has the minimum number of reflections (Sacchi 1997; Selesnick and Bayram 2014; Taylor et al. 1979). The technique creates a matrix of Klauder wavelets and performs calculations while maintaining zero-phase characteristics. This technique maintains only the reflections that have
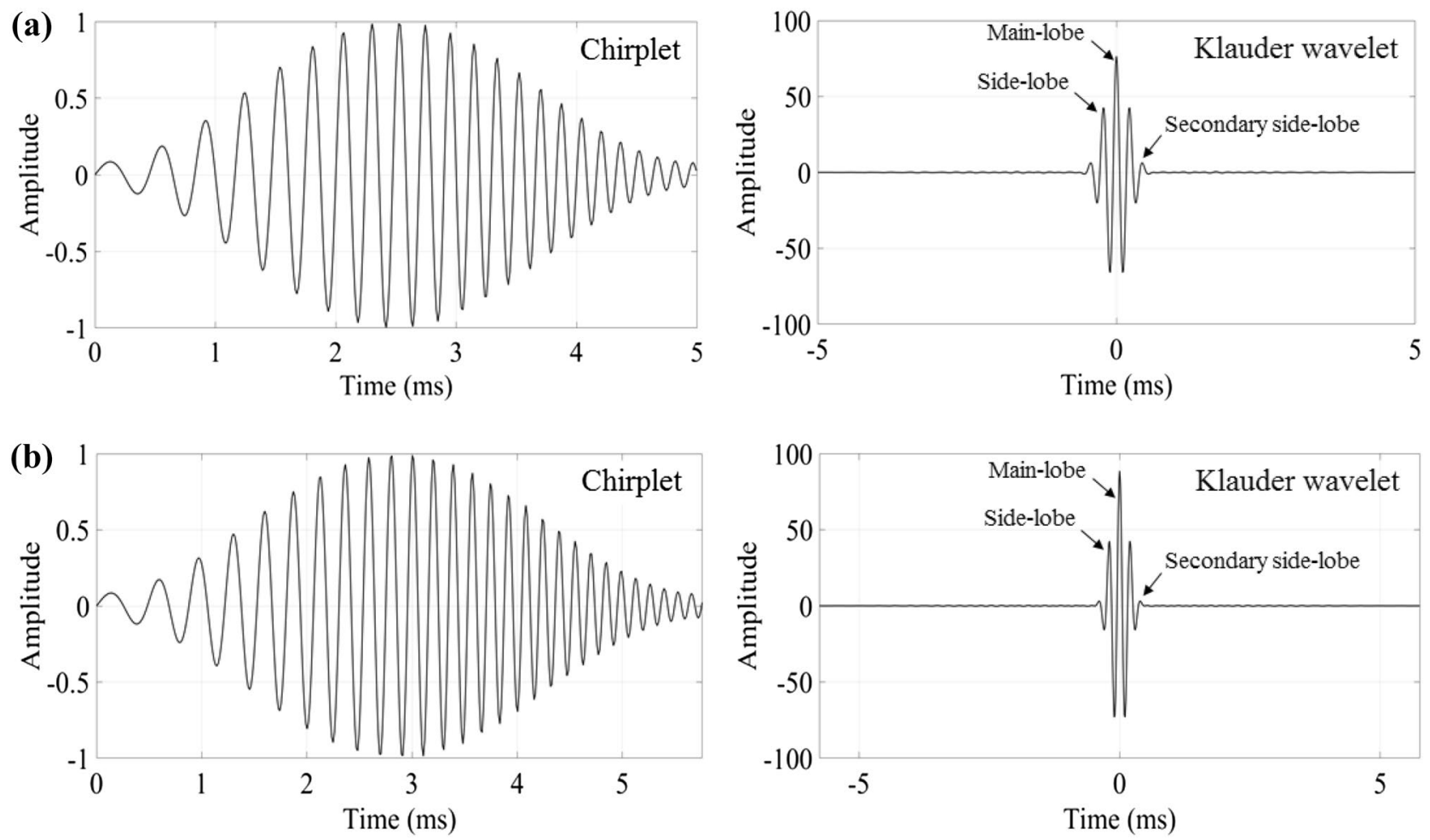

Fig. 6 Artificial chirplet and Klauder wavelet: a using original chirplet signature's variables; $\mathbf{b}$ using optimal chirplet signature's variables 


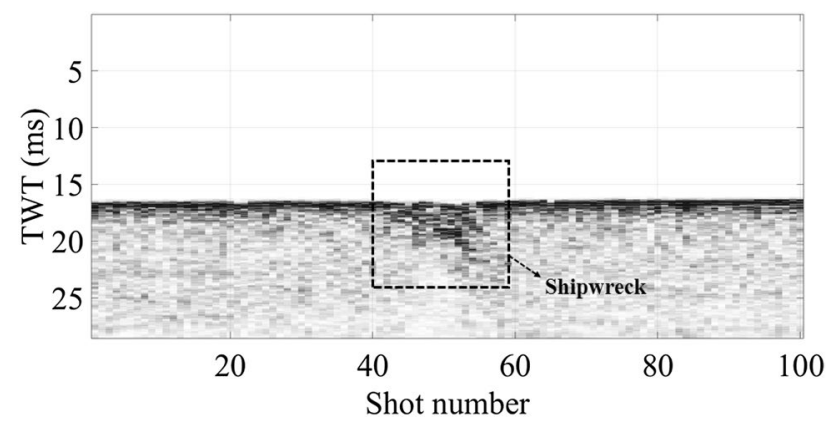

Fig. 7 Post-processed optimal Klauder wavelet section. The dotted rectangle shows the location of the shipwreck

relatively large reflection amplitudes, by directly removing Klauder wavelet components from the Klauder wavelet profile, and simultaneously reducing the reflection values caused by the Klauder wavelet's side-lobe to almost 0 . The equation for this is described in detail in Appendix B of the paper by Taylor et al. (1979).

The appropriate regularization parameter range for deconvolution is 5-20; at 10 . Here, the regularization parameter is a variable related to the amount of pre-whitening. Moreover, the variable density display profile and the wiggle display profile of the reflection coefficient, which is expressed through spikes, were obtained through 20 iterations (Fig. 8). In the wiggle profile, it can be seen that there was a very weak reflection intensity caused by
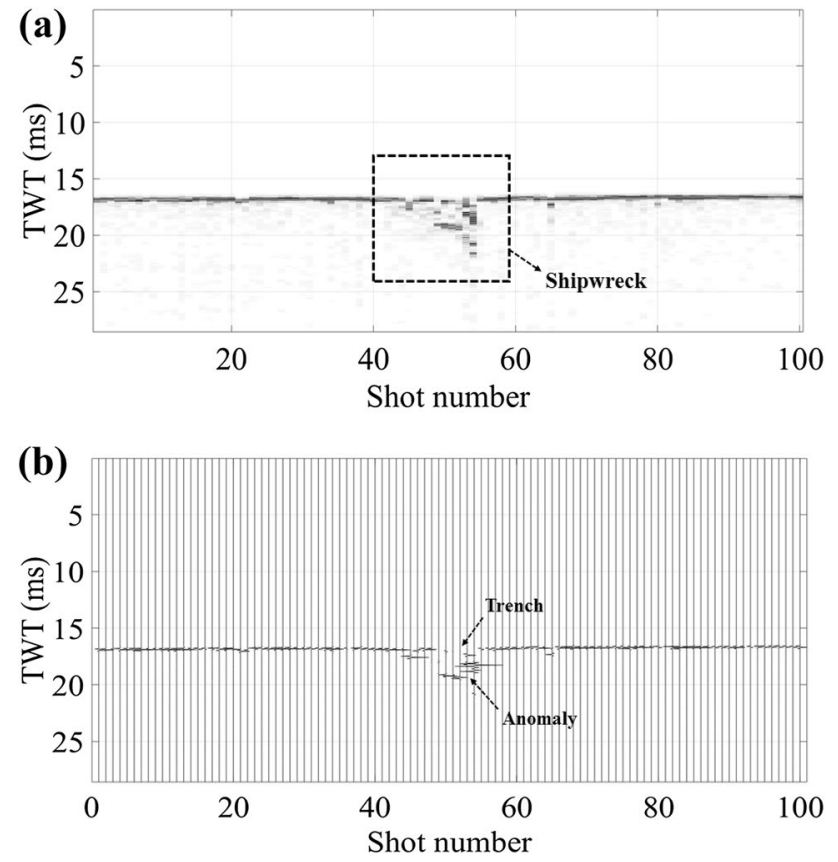

Fig. 8 Sparse-spike deconvolution results. The dotted rectangle shows the location of the shipwreck. a Density section, and $\mathbf{b}$ wiggle section the shipwreck that was exposed by the test pit. This corresponds with studies and experiment results which show that the reflection value is close to zero or negative due to the long-term decay of the wood (Arnott et al. 2005).

\section{Acoustic impedance conversion}

The acoustic impedance conversion used in this study uses the reflection coefficient, which is the ratio of the impedances of the strata on either side of the interface, calculated by sparse-spike deconvolution. The equation is as follows:

$Z_{i+1}=Z_{i} \times\left(1+R_{i}\right) /\left(1-R_{i}\right)$

Here, $Z_{i}$ is the upper stratum's acoustic impedance; $Z_{i+1}$ is the lower stratum's acoustic impedance; $R_{i}$ is the upper stratum's reflection coefficient.

The first layer, which is seawater, was assumed to have a velocity of $1500 \mathrm{~m} / \mathrm{s}$ and a density of $1025 \mathrm{~kg} / \mathrm{m}^{3}$, and therefore, an impedance of $1.53\left(\times 10^{6} \mathrm{~kg} / \mathrm{m}^{2} \mathrm{~s}\right)$ was used. In the acoustic impedance results using the original chirplet, the impedance of the shipwreck was similar to its surroundings, and therefore, was not visually distinct. However, in the acoustic impedance results using the optimal chirplet, the shipwreck area's impedance value was markedly higher, and therefore appeared to be visually distinct (Fig. 9).
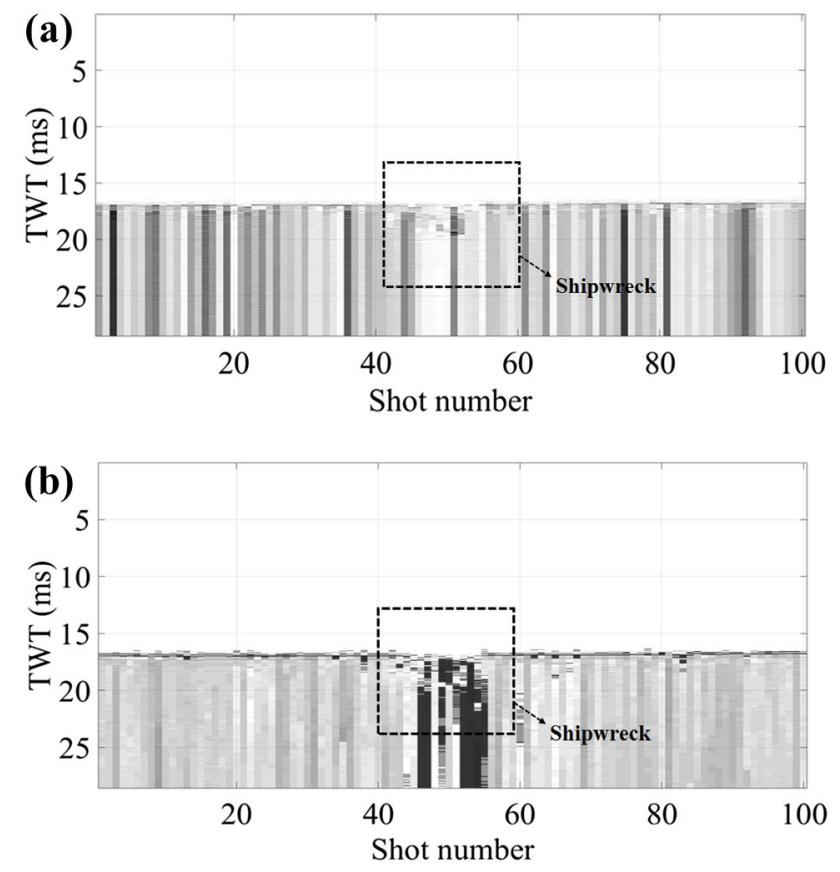

Fig. 9 Acoustic impedance profile: a results using original chirplet; b results using optimal chirplet. The dotted line shows the location of the shipwreck 


\section{Analysis of acoustic impedance results}

The derived acoustic impedance model shows a significantly high impedance value in the area where the shipwreck was located. This impedance value was due to the relics scattered inside and outside the wreck, rather than the decayed wood of the ship itself.

In this section, the impedance results from using the original chirplet on the trace where the shipwreck was located, were compared to those obtained using the optimal chirplet. In addition, the impedance results were verified by comparing them to the impedance results of the study area where iron pots and ceramics were buried in the seabed.

\section{Comparison and analysis of acoustic impedance results}

In this study, acoustic impedance results using the original and optimal chirplets were compared. The acoustic impedance value was based on 50 traces where anomalies were located. Here, there was a test pit on the seafloor, so the impedance of the original seabed surface layer was not recorded.

The acoustic impedance results using the original chirplet showed a low impedance value of $1.44-1.54\left(\times 10^{6} \mathrm{~kg} / \mathrm{m}^{2}\right.$ $\mathrm{s})$ in the anomaly section. Moreover, the bottom part of the anomaly showed an impedance of around 1.49 , which was lower than that of seawater. On the other hand, the acoustic impedance results using the optimal chirplet were 1.25-3.0 $\left(\times 10^{6} \mathrm{~kg} / \mathrm{m}^{2} \mathrm{~s}\right)$ in the anomaly section, and these values indicate a correlation to the iron pots, ceramics, and other debris. Furthermore, the bottom of the anomaly showed a value of around $1.57\left(\times 10^{6} \mathrm{~kg} / \mathrm{m}^{2} \mathrm{~s}\right)$, which is a slightly higher impedance than the seawater. It is assumed that the medium's impedance was slightly higher than seawater

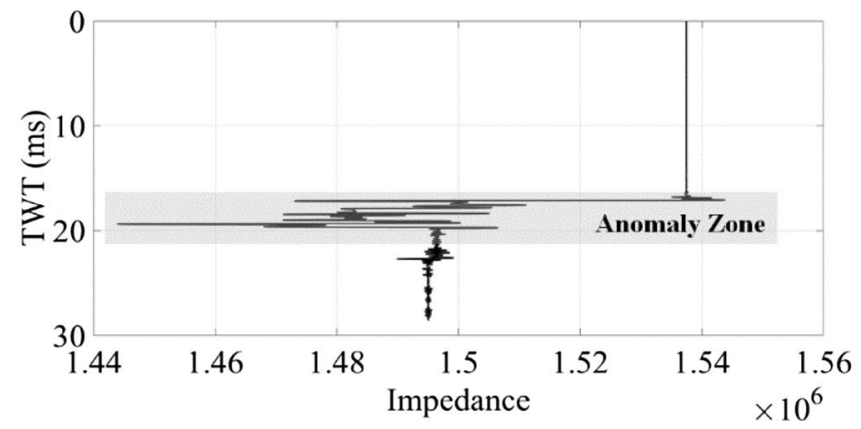

(a) because the sediment environment in the survey area was very soft (Fig. 10).

\section{Verification by comparison with study area acoustic impedance}

Divers inspected the shipwreck and the relics, such as iron pots and ceramics, onsite. Nevertheless, this study has verified its quantitative results through a comparison with a control study area.

The study area is very close to the shipwreck area where iron pots and ceramics are buried $2 \mathrm{~m}$ under the seabed (Fig. 1). In the study area, data processing was performed in the same way using the optimal variables used in this study. The results after completing post-processing (Phase 3) are shown in Fig. 11, where the diffractions from the iron pots and ceramics can be confirmed by sight.

Figure 12 shows the acoustic impedance result values for parts of the study area where groups of iron pots and ceramics were located. The impedances of the study area's seabed surface and media at the bottom parts of anomalies were between 1.5 and $1.7\left(\times 10^{6} \mathrm{~kg} / \mathrm{m}^{2} \mathrm{~s}\right)$. The iron pots showed an impedance of around $2.9\left(\times 10^{6} \mathrm{~kg} / \mathrm{m}^{2} \mathrm{~s}\right)$, and the

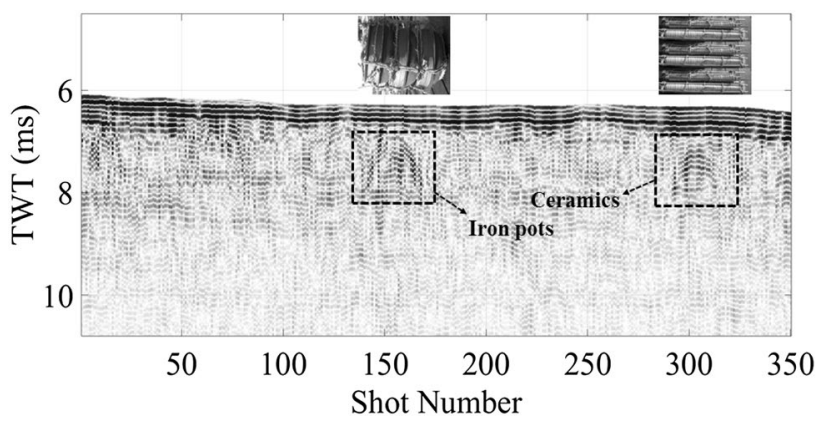

Fig. 11 Results of post-processing (Phase 3) on study area where groups of iron pots and ceramics were buried

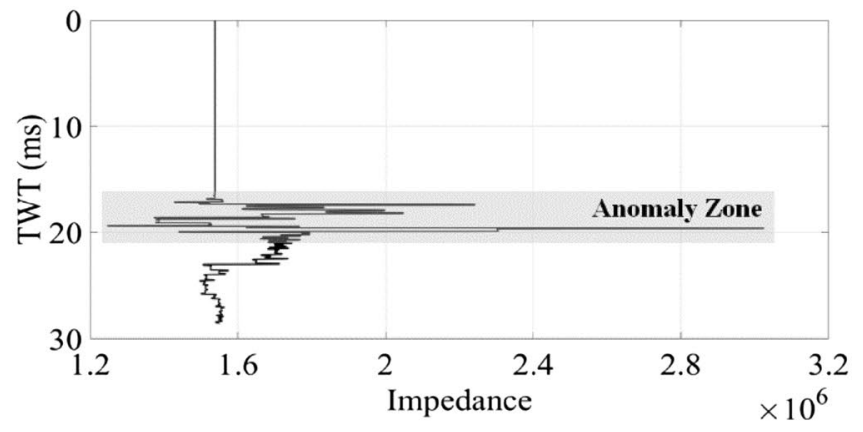

(b)

Fig. 10 Acoustic impedance results of 50 traces located in the anomaly area: a using original chirplet; $\mathbf{b}$ using optimal chirplet. Acoustic section refers to the area where the anomaly is located 

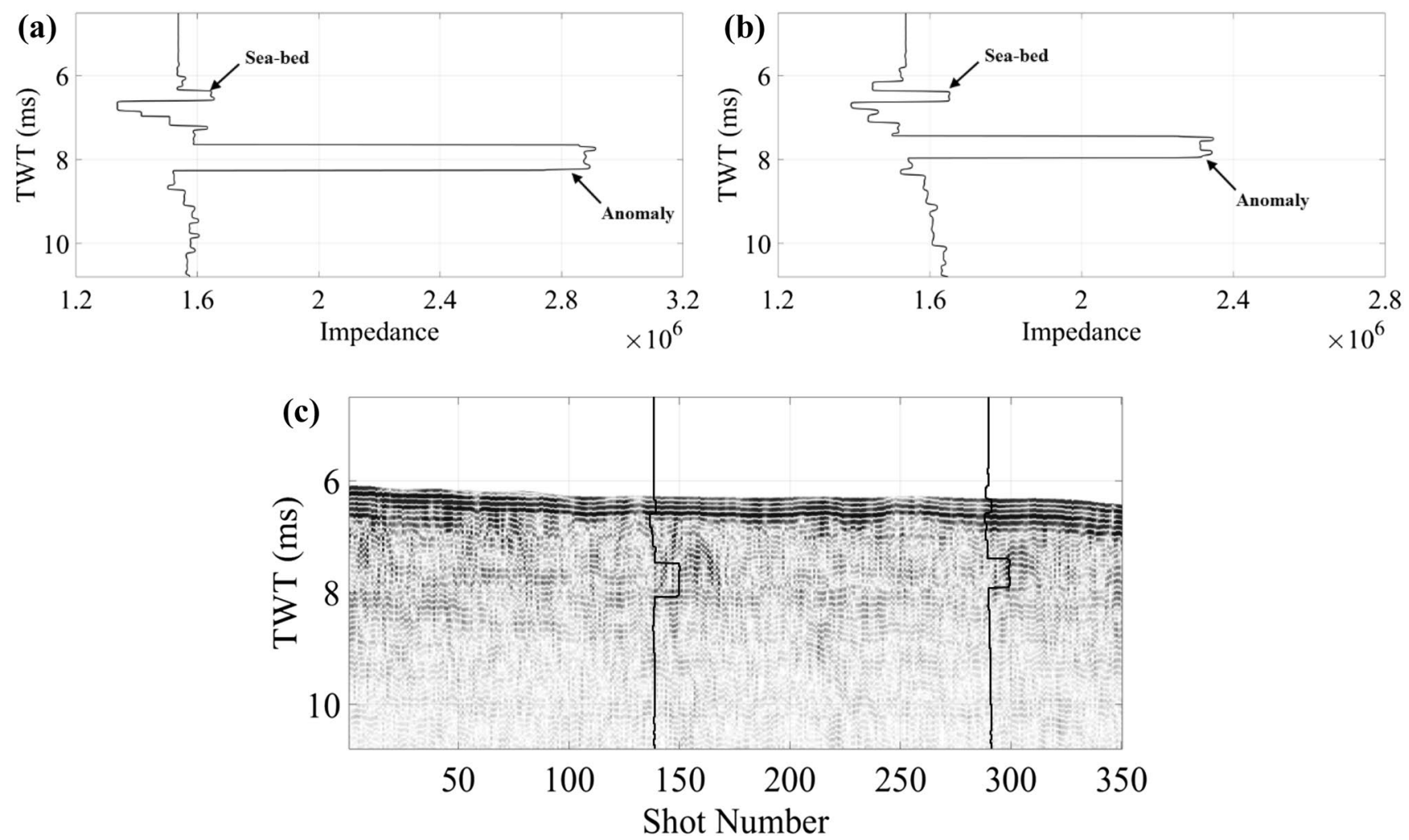

Fig. 12 Study area's acoustic impedance results. a Results of 150 traces where iron post is located; $\mathbf{b}$ results of 300 traces where ceramics are located; and c post-processed Klauder wavelet section and overlapped results

ceramics showed an impedance of around $2.35\left(\times 10^{6} \mathrm{~kg} / \mathrm{m}^{2}\right.$ s), similar to the shipwreck impedance results.

\section{Conclusions}

This study performed sequential data processing, sparsespike deconvolution, and acoustic impedance conversion using an optimal chirplet found in the seabed reflection signal of raw data, rather than the original chirplet set during seismic surveying. In Klauder wavelets, which are autocorrelated chirplets, the optimal chirplet created waveforms that were more spiked than the original Klauder wavelet, by increasing the main-lobe amplitude, decreasing the sidelobe amplitudes, and shortening the time between sidelobes. Based on these results, the location and reflection coefficient intensity can be found more accurately during deconvolution.

In the acoustic impedance results using the original chirplet, anomalies showed very low impedance while the medium at the bottom of the anomalies showed lower impedance than seawater. However, in the acoustic impedance results using the optimal chirplet, the impedance caused by the media and relics generally showed more appropriate values. These results are similar to those found in the study area where iron pots and ceramics were intentionally buried, and therefore, validates the acoustic impedance results.

This study shows that accurate results can only be obtained if the chirplet signature is found in the raw data, because considerable waveform distortion occurs as the chirplet is transmitted through media. Furthermore, the possibility of analyzing quantitative physical properties will increase significantly if a similar process is performed with high frequency SBP sound sources such as a pinger, sparker, or boomer.

Acknowledgements This work was supported by the Basic Research Project "Development of real-time monitoring mobile system for shallow 3D seismic survey" of the Korea Institute of Geoscience and Mineral Resources (KIGAM) funded by the Ministry of Science and ICT of Korea.

OpenAccess This article is distributed under the terms of the Creative Commons Attribution 4.0 International License (http://creativeco mmons.org/licenses/by/4.0/), which permits unrestricted use, distribution, and reproduction in any medium, provided you give appropriate credit to the original author(s) and the source, provide a link to the Creative Commons license, and indicate if changes were made. 


\section{References}

Arnott SHL, Dix JK, Best AI, Gregory DJ (2005) Imaging of buried archaeological materials: the reflection properties of archaeological wood. Mar Geophys Res 26(2-4):135-144

Baradello L (2014) An improved processing sequence for uncorrelated chirp sonar data. Mar Geophys Res 35(4):337-344

Bull JM, Quinn R, Dix JK (1998) Reflection coefficient calculation from marine high resolution seismic reflection (chirp) data and application to and archaeological case study. Mar Geophys Res 20(1):1-11

Gutowski M, Bull J, Henstock T, Dix J, Hogarth P, Leighton T, White $P$ (2002) Chirp sub-bottom profiler source signature design and field testing. Mar Geophys Res 23(5-6):481-492

Gutowski M, Bull JM, Dix JK, Henstock TJ, Hogarth P, Hiller T, Leighton TG, White PR (2008) 3D high-resolution acoustic imaging of the sub-seabed. Appl Acoust 69(3):262-271

Henkart P (2006) Chirp sub-bottom profiler processing - a review. Sea Technol 47(10):35-38

Kim YJ (2014) Field applications of 2-D/Pseudo 3-D chirp raw data for the high-resolution sub-bottom profiling, Ph.D. Thesis, Korea Maritime and Ocean University

Kim YJ, Koo NH, Cheong S, Kim JK, Chun JH, Shin SR, Riedel M, Lee HY (2016) A case study on pseudo 3-D Chirp sub-bottom profiler (SBP) survey for the detection of a fault trace in shallow sedimentary layers at gas hydrate site in the Ulleung Basin, East Sea. J Appl Geophys 133:98-115

LeBlanc LR, Mayer L, Rufino M, Schock SG, King J (1992) Marine sediment classification using the chirp sonar. J Acoust Soc Am 91(1):107-115
Mann S, Haykin S (1995) The Chirplet transform: physical considerations. IEEE Trans Signal Process 43(11):2745-2761

Quinn R, Bull JM, Dix JK (1998) Optimal processing of marine highresolution seismic reflection (Chirp) data. Mar Geophys Res 20(1):13-20

Sacchi DM (1997) Reweighting strategies in seismic deconvolution. Geophys J Int 129(3):651-656

Schwamborn GJ, Dix JK, Bull JM, Rachold V (2002) High-resolution seismic and ground penetrating radar-geophysical profiling of a Thermokarst lake in the Western Lena Delta, Northern Siberia. Permafr Periglac Process 13(4):259-269

Selesnick IW, Bayram I (2014) Sparse signal estimation by maximally sparse convex optimization. IEEE Trans Signal Process 62(5):1078-1092

Stevenson IR, McCann C, Runciman PB (2002) An attenuation-based sediment classification technique using Chirp sub-bottom profiler data and laboratory acoustic analysis. Mar Geophys Res 23(4):277-298

Taylor HL, Banks SC, McCoy JF (1979) Deconvolution with the L1 norm. Geophysics 44(1):39-52

Publisher's Note Springer Nature remains neutral with regard to jurisdictional claims in published maps and institutional affiliations. 